\title{
MODELAÇÃO ESPACIAL DA ADEQUABILIDADE DE HABITAT A ESPÉCIES INVASORAS: O CARPOBROTUS EDULIS EM TERRENO NÃO DUNAR*
}

CÉSAR CAPINHA ${ }^{1}$

\begin{abstract}
Resumo - A modelação de habitats potenciais para espécies nativas encontra-se já bastante divulgada, sendo numerosos os exemplos existentes. Uma abordagem metodológica semelhante, mas com suporte teórico diferente é a sua realização para espécies exóticas invasoras. Este trabalho apresenta uma metodologia de modelação espacial da adequabilidade de habitat ao Chorão-de-praia (Carpobrotus edulis), uma planta exótica invasora, na Serra do Bouro em Portugal. Para isso foi efectuado um levantamento de presenças desta espécie, optimizado pela sua persistência e manifestação de invasão. Conjuntamente foram criados registos de pseudo-ausência, de forma a permitir a utilização de uma regressão logística, que se considera apresentar melhores resultados preditivos do que modelos de presença apenas. É proposta uma metodologia de validação, que conjuga a área mínima de adequabilidade máxima com a quantidade de registos de validação correctos. Para esta, e considerando-se um limiar de probabilidade de 0,7 , obtiveram-se $80 \%$ de presenças correctas para uma área correspondente a apenas $8 \%$ da área total. $\mathrm{O}$ abandono agrícola foi identificado como factor potenciador da sua expansão, em conjugação com reduzidas distâncias à linha de costa e áreas sujeitas a erosão moderada. Inversamente, áreas interiores, ou com elevados declives e vertentes extensas, revelaram uma reduzida adequabilidade para a espécie.
\end{abstract}

Palavras-chave: Espécies invasoras, modelação, SIG, adequabilidade de habitat.

\begin{abstract}
Spatial MOdelling of HABitat SUITABility FOR INVASIVE SPECIES: THE CARPOBROTUS EDULIS IN A NON-DUNE AREA. The use of habitat modelling for exotic invasive species can be extremely useful for identifying their potential impacts and for assisting in the design of eradication strategies. Even though the latter builds on theoretical assumptions that are quite different from those involved in the modelling of the habitat of native species, these two modelling methods are in fact quite similar. This article presents a habitat suitability modelling framework for Carpobrotus edulis, an alien invader plant in Serra do Bouro, Portugal. Several land surveys have been carried out in the study area in order to record the presence of this plant. The criteria for recording a presence were that the plant did not show any signs of weakness and
\end{abstract}

* Recebido: 5/03/2008. Revisto: 30/09/2008. Aceite: 24/10/2008.

1 Centro de Estudos Geográficos da Univ. de Lisboa. E-mail: cesarcapinha@hotmail.com 
that there were mat formations covering at least $5 \mathrm{~m}^{2}$. Pseudo-absences were also obtained in a completely random way. The model was calibrated using a binary logistic regression. The performance of this model usually considered superior to that of models that rely on presence data only. Additionally, an evaluation technique based on the minimum area of higher adequacy is also presented. This technique assumes that, for a given probability threshold, model performance is higher whenever it has the same number of correct presences for a smaller predicted area. Using a 0.7 probability threshold, the model correctly predicted $80 \%$ of the total presences using only $8 \%$ of the study area. The model suggests that the main factor contributing to the expansion of Carpobrotus edulis has been the abandonment of agriculture in the study area. In addition, proximity to the shoreline and above-average erosion potential in the study area both seem to benefit the plant's expansion. Conversely, steeper and longer slopes, and greater distances from the shoreline, were found to be significant contributors to the plant's absence.

Key words: Invasive species, modelling, GIS, habitat suitability.

Résumé - ModélisATION SPATIALE DE L'AdÉQUATION DE L'HABITAT AUX ESPÈCES ENVAHISSANTES: LE CAPROBROTUS EDULIS EN TERRAIN NON DUNAIRE. La modélisation d'habitats potentiels pour les espèces indigènes est aujourd'hui bien connue et les exemples en sont nombreux et utiles. Son application aux espèces exotiques envahissantes implique une méthodologie analogue, mais avec un support théorique différent. On présente un projet de modélisation spatiale de l'adéquation de la notion d'habitat à la Figue marine (Caprobrotus edulis), plante exotique envahissante de la Serra de Bouro (Portugal). On a pour cela enregistré les lieux de présence de cette espèce, en notant aussi sa persistance et les marques d'invasion. On a créé un registre de pseudoabsence, pour permettre l'utilisation d'une régression logistique, qui donne de meilleurs indices prédictifs qu'un simple modèle de présence. On propose une méthode de validation, qui associe une aire minimale d'adéquation maximale avec le nombre d'enregistrements de validation corrects. En admettant un seuil de probabilité de 0,7, on obtient $80 \%$ de présences correctes pour une surface de seulement $8 \%$ de l'aire totale. Les facteurs favorables à l'expansion de l'espèce sont l'abandon des cultures, la proximité du littoral et une érosion modérée. Par contre, les régions intérieures et fortement accidentées lui sont peu favorables.

Mots-clés: Espèces envahissantes, modélisation, SIG, adéquation de l'habitat.

\section{INTRODUÇÃO}

As invasões biológicas por parte de espécies exóticas são reconhecidas como uma das principais ameaças à biodiversidade (Williamson, 1996). Muitos dos seus efeitos negativos também se fazem sentir de forma directa nas actividades económicas (Pimentel et al., 2000; Evans, 2003) e até na saúde humana (Juliano e Lounibos, 2005; Qiau-Ping et al., 2007). A tendência para um comércio cada vez mais global é tida como um dos principais vectores para a crescente introdução de espécies não nativas, quer de forma intencional quer inadvertidamente. A redução de fronteiras tem possibilitado o transporte de muitas destas espécies 
a grandes distâncias dos locais onde sofreram os processos de especiação (Perrings et al., 2005). O comportamento populacional destas espécies nos novos locais está geralmente revestido de elevada complexidade podendo, ou não, levar a comportamentos invasores. Pese embora o conhecimento generalizado de que a solução para este problema passa sobretudo pela prevenção, muitas das acções acabam por ter como objectivo a mitigação de impactes, sendo apenas implementadas após a manifestação inequívoca de comportamentos invasores e dos seus efeitos.

O estudo das invasões biológicas tem tido, na sua componente espacial, uma das principais linhas de estudo desde há várias décadas (Hastings et al., 2005). A abordagem ao estudo espacial destes fenómenos tem derivado várias temáticas, tais como a cartografia de áreas invadidas, a adequabilidade de habitats ou a análise de padrões de dispersão. Estando intimamente ligadas entre si, estas abordagens têm usado várias ferramentas e metodologias, sendo de destacar o contributo recente dos Sistemas de Informação Geográfica (SIG) e a sua integração com diversos modelos estatísticos preditivos. No que diz respeito à obtenção de modelos que quantifiquem o grau de habitabilidade de determinado território para uma espécie, que é objecto de estudo deste trabalho, são vários os exemplos existentes. Estes, ainda assim, representam um número bastante menos expressivo que outros metodologicamente semelhantes como a modelação de habitats potenciais de espécies nativas. A modelação de habitats potenciais frequentemente utilizada nas áreas da biologia da conservação e da macroecologia para espécies autóctones apresenta inúmeros casos de aplicação, sendo as diversas metodologias possíveis para a sua realização objecto de vários estudos (Guisan e Zimmerman, 2000), assim como a avaliação dos seus desempenhos preditivos (Segurado e Araújo, 2004; Tsoar et al., 2007).

A modelação espacial da adequabilidade para espécies invasoras tem-se baseado sobretudo nestas ferramentas e metodologias (Peterson e Vieglais, 1999; Haltuch et al., 2000; Underwood et al., 2004; Ficetola et al., 2007); no entanto, um aspecto essencial diverge da abordagem geral destes modelos e da modelação de habitats potenciais para espécies exóticas invasoras, que se prende com o pressuposto de que as espécies nativas se encontram em equilíbrio com o ecossistema (Guisan e Zimmerman, 2000). O facto de o período temporal de interacção com o meio ser muitas vezes reduzido, leva a que em muitos casos as espécies invasoras não tenham ainda sido confrontadas com todas as adversidades que este lhes pode imputar, reduzindo as suas áreas de colonização. Pelo contrário, não se tendo ainda dispersado pela totalidade da área de estudo pode haver locais ainda mais propensos à ocupação do que os actualmente invadidos, sem que a espécie aí ocorra. Estas situações levam a que a distribuição da espécie, aquando da sua análise no terreno, possa não reflectir de forma correcta o seu verdadeiro habitat potencial. Neste sentido, os levantamentos de campo de presença ou ausência ao longo da área de estudo constituem um passo de elevada sensibilidade na construção deste tipo de modelos para espécies invasoras. 
Um outro aspecto digno de atenção na realização de modelos de adequabilidade de habitat para espécies exóticas invasoras prende-se com o facto da variável resposta dos modelos estatísticos ser do tipo presença/ausência ou de presença apenas. O levantamento de ausências de espécies invasoras é complexo, pois enquanto as presenças podem ser optimizadas pela análise da persistência dos indivíduos, ou da própria manifestação do processo de invasão, o levantamento de ausências reveste-se de maiores incertezas, sendo bastante mais duvidoso inferir que a não ocorrência da espécie invasora se deve à falta de condições necessárias nesse local. A necessidade de muitos modelos estatísticos por dados do tipo presença-ausência, faz com que sejam tidos, numa abordagem inicial, como inadequados para trabalhar com distribuições de espécies que não são nativas. Este aspecto tem levado a que os modelos estatísticos maioritariamente utilizados nesta área sejam apenas baseados em dados de presença (Peterson et al., 2001; Cawley, 2003; Ficetola et al., 2007). Contudo, estes modelos apresentam regra geral os desempenhos mais baixos nas predições da distribuição de espécies nativas, quando comparados com métodos que utilizam dados do tipo presença/ausência (Segurado e Araújo, 2004; Elith et al., 2006), o que leva a crer que a inclusão de dados de ausência na modelação de espécies invasoras contribua também para a sua melhoria preditiva.

Este trabalho pretende apresentar uma metodologia de modelação espacial da adequação do habitat para a planta invasora Carpobrotus edulis numa área litoral designada de Serra do Bouro utilizando para isso registos de presenças e pseudoausências. Ao invés de serem interpretados como uma verdadeira ausência de indivíduos, os registos de pseudo-ausência pretendem agir como uma amostra das características gerais da área (Ferrier et al., 2002). Brotons et al., (2004), aplicaram este método à modelação da distribuição de espécies de aves nativas para as quais apenas eram conhecidos registos de presença, tendo sido notória a sua melhoria preditiva relativamente ao modelo proveniente da utilização de dados de presença apenas. A metodologia de levantamentos de campo e forma de avaliação de desempenho do modelo são também discutidas com vista à optimização dos métodos de modelação espacial de adequabilidade de habitat para espécies invasoras não nativas. Por fim, são discutidos os resultados com vista à obtenção de um melhor conhecimento das áreas mais adequadas para esta espécie e do seu potencial invasor.

\section{CARACTERIZAÇÃO DA ÁREA DE ESTUDO E DA ESPÉCIE INVASORA}

\section{1. Área de estudo: Serra do Bouro}

A área de estudo corresponde à Serra do Bouro, um alto-relevo costeiro de baixa altitude (máx.: 162m), com uma orientação SW-NE situada no concelho de Caldas da Rainha (fig. 1). O seu limite, marcado a Oeste por uma linha de costa rochosa, composta essencialmente por arribas abruptas, não encontra no quadrante 
oposto uma fronteira claramente definida. A sua demarcação para este estudo seguiu as áreas de maior declive, com altitudes entre 40 e $60 \mathrm{~m}$. A área total de estudo é de 1645 hectares.

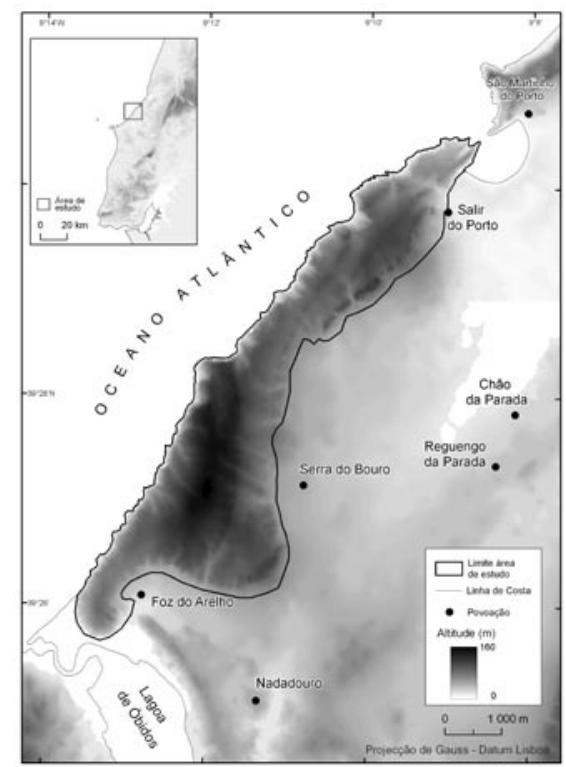

Fig. 1 - Enquadramento espacial da área de estudo Fig. 1 - Location of the study area

A cobertura de solo aqui existente está em grande parte relacionada com a acção humana, seja por actividade agrícola extensiva, por uso florestal ou por uso urbano. A utilização agrícola é dominante e ocorre sobretudo nas áreas aplanadas de topo e ao longo das áreas com declives mais suaves da encosta Este. A extensão destas áreas foi outrora maior, reconhecendo-se na Serra muitos locais com traços visíveis desta actividade já abandonada. Muitos destes correspondem agora a locais onde predomina o coberto herbáceo, ou estádios de transição deste para arbustivo. As áreas florestadas apresentam algumas manchas dispersas ao longo da Serra mas são sobretudo representativas junto à linha de base da encosta nascente. Existem ainda alguns locais onde a acção humana não é tão visível, correspondendo nestes casos a manchas de vegetação arbustiva compostas essencialmente por Ulex sp. e Erica sp.. A sua distribuição é no entanto dispersa, tendo a sua representatividade máxima no extremo Norte da Serra.

O substrato geológico é composto por calcários, arenitos argilosos, margas e argilas do período Jurássico. Os solos são maioritariamente argilosos, existindo ainda assim vários locais onde a fracção arenosa é dominante. Estes solos arenosos encontram-se nas áreas aplanadas de topo sujeitas a acção 
humana (agrícola e florestal) e próximo das extremidades Norte e Sul da Serra, onde as areias são transportadas das praias adjacentes por acção do vento. Dada a reduzida extensão da área, o contraste climático é proveniente essencialmente das variações topográficas, sendo de destacar a acção do vento. A proximidade ao mar faz com que esta seja uma área bastante fustigada pelo vento, carregado com elevados teores de sal. A sua acção é ainda acentuada pela orientação dominantemente perpendicular aos ventos de NW, e pelos declives abruptos das arribas.

\section{A espécie invasora: Carpobrotus edulis}

O Carpobrotus edulis (L.) N.E. Br., vulgarmente conhecido por Chorão-dapraia é uma planta perene com capacidade de formar extensos tapetes homogéneos, competindo directamente com as espécies nativas e impedindo o seu restabelecimento. Esta planta, nativa da África do Sul (Schmalzer e Hinkle, 1987 citando Washburn e Frankie, 1985), foi introduzida em muitos locais como ornamental e para fixação de dunas, existindo registos da sua existência em locais da Europa Ocidental já desde o séc. XIX (Arguimbau et al., 2004). Embora a sua posse e cultivo se encontrem proibidos pelo sistema legal português (Decreto-lei 565/99 de 21 de Dezembro), são vários os jardins públicos e privados na área de estudo a apresentar esta planta, sendo visíveis em seu redor muitas áreas naturais invadidas.

As informações acerca das preferências ecológicas da espécie são relativamente esparsas, sendo várias delas baseadas em estudos para locais onde são introduzidas. Esta característica faz com que algumas das preferências assinaladas sejam de carácter menos generalizável, pois as espécies invasoras podem apresentar comportamentos de invasão diferentes para locais de introdução distintos (Sakai et al., 2001). Alguns factores preferenciais são, no entanto, habitualmente referidos: solos arenosos e bem drenados (Schmalzer e Hinkle, 1987; Gilman, 1999), locais não sujeitos a frios excessivos (Schmalzer e Hinkle, 1987; DiTomaso e Healy, 2006), boa exposição solar (Gilman, 1999) e áreas sujeitas a perturbações (Schmalzer e Hinkle, 1987; DiTomaso e Healy, 2006).

\section{MÉTODOS}

\section{Amostragem}

Os levantamentos das presenças da espécie foram efectuados directamente no terreno, de forma exaustiva. A sua localização foi registada como um ponto de coordenadas conhecidas com recurso a um GPS com correcção diferencial, permitindo auferir de um erro posicional máximo de 3 metros. As áreas urbanas não foram consideradas devido ao papel humano directo na presença destas espécies para estes locais. 
Durante este levantamento, tendo em conta a possibilidade de existência de curtos espaços temporais de interacção indivíduos-meio, foram apenas registadas presenças que evidenciassem uma forte vitalidade das plantas e sobretudo manifestações de invasão nítidas. No que diz respeito à sua vitalidade não foram registadas as presenças em que os indivíduos se apresentavam com claros indícios exteriores de debilidade, como coloração atípica ou emurchecimento. Relativamente às manifestações de invasão, ainda que as presenças fossem constituídas por plantas aparentemente saudáveis, só foram considerados os casos em que havia coberturas com pelo menos $5 \mathrm{~m}^{2}$, tentando-se com isso excluir aquelas que, embora existentes na área, não reuniam as condições necessárias à ocupação das áreas envolventes. Por fim, e considerando a resolução espacial do modelo (10 metros), optou-se por estabelecer esse valor como a distância mínima entre pontos recolhidos de forma a evitar a duplicação de registos. No total foram recolhidos 144 pontos de presença, tendo-se optado por reter 30 destes para a avaliação do modelo por validação cruzada.

Além dos registos de presenças foram também obtidos registos de pseudoausências. A utilização destes registos pretende funcionar sobretudo como uma representação das propriedades gerais da área de estudo. A sua utilização, além de permitir alargar o leque de escolha dos modelos de integração estatística, apresenta em vários trabalhos resultados preditivos superiores aos métodos que utilizam apenas dados de presença (Engler et al., 2004; Elith et al., 2006). A obtenção destas pseudo-ausências pode ser realizada, quer de forma aleatória, quer por extracção direccionada, após uma análise exploratória inicial dos dados de presença (Engler et al., 2004). A sua extracção, além de procurar transmitir as características das variáveis representativas da área, deve também ter em conta o número de registos de presença disponíveis caso o método de integração estatística a utilizar seja sensível à desigualdade no número de registos para cada um dos conjuntos. A possibilidade de exploração inicial dos dados de presenças para guiar o processo de escolha é mais favorável para estudos com espécies nativas em que, teoricamente, o conjunto das suas presenças tem menores probabilidades de enviesamento por a espécie se encontrar em equilíbrio (ou pseudoequilíbrio) com o ecossistema. Para este trabalho, tendo em consideração os pressupostos anteriores, optou-se por realizar uma selecção inteiramente aleatória dos locais num número idêntico ao dos registos de presença $(n=114)$, tendo-se utilizado uma função apropriada no software SIG ILWIS 3.4 Open (Ilwis, 2007). A carta com as presenças, pseudo-ausências e registos de validação é visível na figura 2.

\section{Modelação do índice de adequabilidade de habitat}

As variáveis ambientais a integrar neste tipo de modelos são tidas como um dos principais factores passíveis de condicionar o seu desempenho. A obtenção de variáveis com maior potencial preditivo, baseadas no conhecimento préexistente dos factores que interferem na distribuição das espécies em questão, é 
tida como um dos grandes passos a dar na modelação de habitats potenciais (Araújo e Guisan, 2006; Tsoar et al., 2007). Considerando este pressuposto, as variáveis escolhidas para integrar o modelo foram, dentro das possibilidades existentes, quer de informação de base, quer de técnicas de modelação conhecidas, aproximando-nos tanto quanto possível dos factores geralmente tidos como influentes na distribuição do C. edulis. Assim, foram considerados dois tipos de variáveis como indicadores das características do terreno: um primeiro relativo à capacidade de transporte de fluxos à superfície e consequente erosão, que inclui o Factor LS (factor que conjuga o declive e a extensão das vertentes), o STI (índice de capacidade de transporte de sedimentos) e o SPI (índice de força de transporte de fluxo); um segundo tipo referente à capacidade de acumulação de água representado pelo índice TWI (índice composto de encharcamento). Para caracterizar os aspectos climáticos foram consideradas duas variáveis: a radiação solar potencial e a distância à linha de costa (no sentido generalista de que transmite a variação da intensidade do vento e a sua acção directa sobre a espécie, mas também a propensão à formação de geadas). Como variáveis representativas do distúrbio a que as áreas se encontram sujeitas foi considerado um modelo do uso de solo com as seguintes classes: herbáceo dominante; arbustivo dominante; agrícola e floresta. A ausência de informação de base actual para a extracção desta informação fez com que este último fosse extraído através da interpretação de fotografias aéreas à escala 1:10 000, de 2005, ou seja, cerca de três anos anteriores à data dos levantamentos. Na tentativa de diminuir o impacto

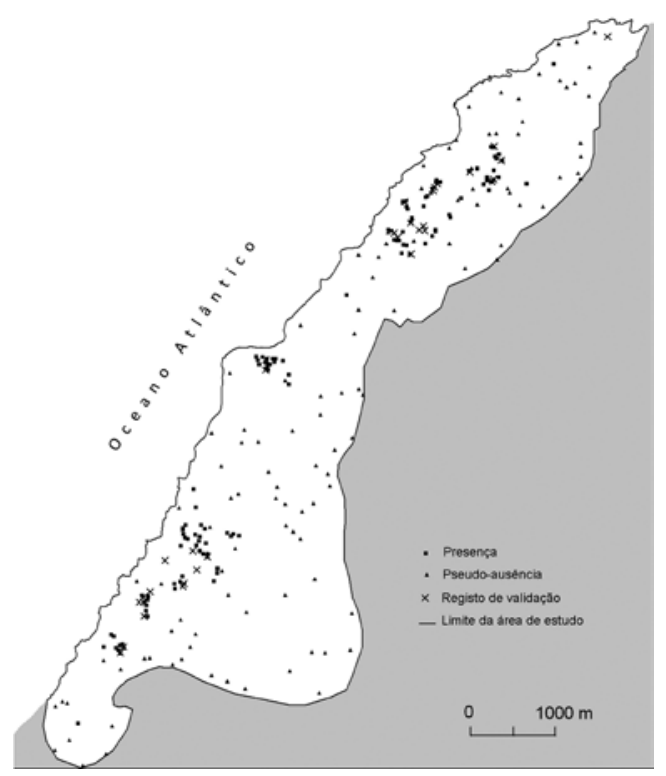

Fig. 2 - Localização dos registos de presença, pseudo-ausência e registos de validação

Fig 2 - Location of the presence, pseudo-absence and validation records 
de possíveis alterações entretanto ocorridas, este modelo foi transformado num conjunto de modelos derivados, correspondendo cada um destes a uma classe de uso distinta contendo o valor da sua cobertura em percentagem para uma matriz de vizinhança de $3 \times 3$ células $\left(900 \mathrm{~m}^{2}\right)$. Esta transformação também é tida como uma tentativa de integrar no modelo, além da informação relativa ao tipo de uso existente, o que ocorre nas suas proximidades, caracterizando de alguma forma a sua heterogeneidade.

O modelo estatístico utilizado neste trabalho foi uma regressão logística binomial. Este modelo apresenta algumas vantagens para um trabalho deste tipo sendo extensivamente utilizado na modelação de habitat potenciais (AlvarezArbesú e Felicísimo, 2002). Dentro das suas características há que destacar o facto de utilizar como variável-resposta dados dicotómicos (presença/ausência) e permitir a exploração do contributo de cada uma das variáveis na construção do modelo. Além disso, é de fácil integração em SIG, com base em dados matriciais, através da aplicação directa da sua equação:

$$
P=\frac{\exp \left(\beta_{0}+\beta_{1} x_{1}+\beta_{2} x_{2} \ldots \ldots .+\beta_{n} x_{n}\right)}{1+\exp \left(\beta_{0}+\beta_{1} x_{1}+\beta_{2} x_{2} \ldots \ldots+\beta_{n} x_{n}\right)}
$$

em que $p$ é a probabilidade de ocorrência do evento (adequabilidade ao $C$. edulis no presente caso), $\beta_{0}$ é um valor constante, $\beta_{i}$ os coeficientes da regressão e $X_{i}$ as variáveis independentes.

Para impedir a existência de multicolinearidade entre as variáveis a integrar o modelo, que pode afectar as suas predições, foi calculado o factor de variância-expansão (VIF) através da integração das variáveis numa regressão linear múltipla. Na tabela de saída desta análise não se identificaram relações de multicolinearidade consideradas elevadas (VIF $>5$ ). Além desta foi também calculada uma matriz de correlações de Pearson, de forma evitar redundâncias nos dados de entrada. Desta análise deduziu-se a eliminação da variável STI, por apresentar pelo menos uma correlação forte $( \pm 0,65$; $p<0,05)$ e por se considerar as variáveis com as quais se correlaciona mais facilmente interpretáveis no contexto do trabalho.

A regressão linear binomial foi aplicada utilizando o método de adição passo-a-passo forward LR (Likelihood Ratio). Este método consiste na adição progressiva das variáveis independentes com maior contribuição para o modelo baseado no teste de Rao's. A sua exclusão é determinada por uma reduzida contribuição obtida pela razão de verosimilhança. Este método constitui-se como o preferível dentro das possibilidades existentes para um processo passo-a-passo (Garson, 2007). O último passo do modelo (6. ${ }^{a}$ iteração) apresentou para o teste de Hosmer-Lemeshow um valor não significativo $(>0,05)$ como é idealmente pretendido. Para um valor de 0,5 como limiar de probabilidade o modelo obteve uma percentagem de classificações correctas de 80,7\% (77,2 \% ausências; 84,2\% presenças). $\mathrm{O}$ quadro 1 apresenta um resumo dos parâmetros finais obtidos. 
Quadro I - Parâmetros da regressão e análises associadas

Table 1 - Regression coefficients and associated analyses

\begin{tabular}{lccccc}
\hline Variável & Parâmetro $\boldsymbol{\beta}$ & Erro padrão & Teste de Wald & Significância & $\operatorname{Exp(\beta )}$ \\
\hline Distância à linha de Costa & $-0,004$ & 0,001 & 24,25 & $\mathrm{p}<0,001$ & 0,996 \\
SPI & $-0,174$ & 0,049 & 12,76 & $\mathrm{p}<0,001$ & 1,119 \\
Factor LS & $-0,512$ & 0,114 & 20,01 & $\mathrm{p}<0,001$ & 0,599 \\
\% Herbáceo dominante & 0,248 & 0,060 & 17,06 & $\mathrm{p}<0,001$ & 1,282 \\
\% Agrícola & 0,132 & 0,064 & 4,21 & $\mathrm{p}<0,001$ & 1,142 \\
Constante & 0,789 & 0,429 & 1,57 & $\mathrm{p}<0,005$ & 2,201 \\
\hline
\end{tabular}

As variáveis consideradas pelo modelo final foram reduzidas para cinco: distância à linha de costa; SPI; Factor LS; percentagem de área com cobertura herbácea dominante e percentagem de área com uso agrícola. A análise do tipo de relação entre as variáveis independentes e a ocorrência da planta pode ser efectuada pela análise dos parâmetros obtidos. A existência de um parâmetro $\beta$ positivo (SPI; \% Herbáceo dominante; \% Agrícola) indica que valores mais elevados da variável a que corresponde contribuem no sentido de maior adequabilidade, enquanto para um valor negativo (Distância à costa; Factor LS) esta será menor. Uma forma mais directa de interpretar o tipo de efeito que as variáveis independentes produzem sobre o fenómeno de estudo é através da análise do expoente de cada parâmetro $\beta$. Este indica a razão de probabilidade da presença em função da alteração de uma unidade da variável independente para uma situação em que o valor das restantes variáveis se mantém.

Assim, pode-se identificar um valor muito próximo de 1 para a variável distância à linha de costa, o que parece indicar a independência desta relativamente à adequabilidade ou não para a espécie. Três factores surgem com uma razão de probabilidade relativamente próximas, SPI $(1,119)$, percentagem de herbáceo dominante $(1,282)$ e percentagem de cobertura agrícola $(1,142)$, indicando que o aumento dos seus valores contribui para a ocorrência de C. edulis, ainda que de uma forma pouco acentuada para incrementos reduzidos. O Factor LS apresenta a razão de probabilidade mais baixa $(0,599)$ indicando um forte decréscimo da probabilidade de adequação com o aumento do valor deste factor.

Os parâmetros obtidos foram utilizados para modelar espacialmente a probabilidade de adequação de habitat para o $C$. edulis através da aplicação da equação da regressão logística (Eq. 1) à informação matricial. O modelo resultante é visível na figura 3 . 


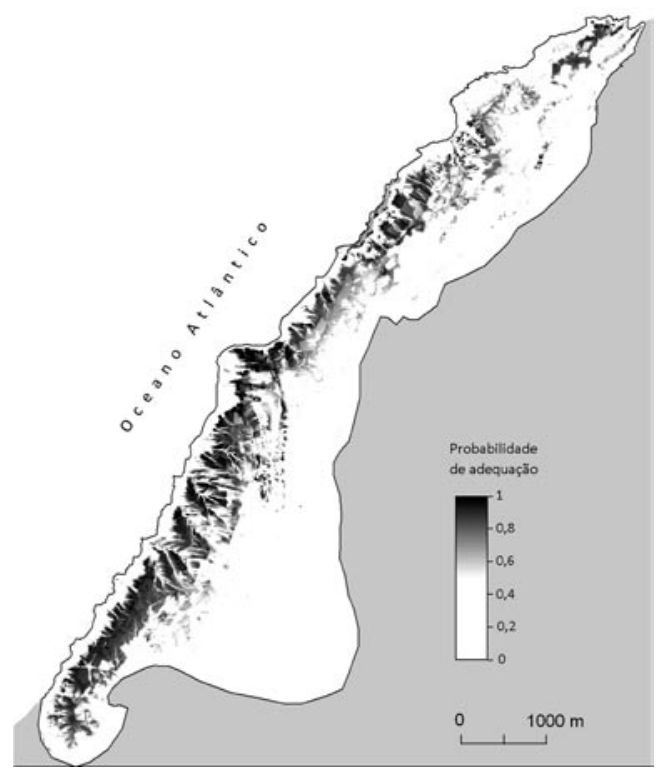

Fig. 3 - Probabilidade de adequação de habitat para o Carpobrotus edulis na Serra do Bouro Fig. 3 - Habitat suitability index for Carpobrotus edulis at Serra do Bouro

\section{Avaliação do modelo}

A avaliação dos resultados espacialmente extrapolados com base em modelos calibrados apenas com dados de presença ou conjugados com pseudo-ausências é bastante menos directa do que para os modelos obtidos a partir de dados de presença/ausência e necessita de maior investigação (Pearce e Boyce, 2006). Quando são utilizados dados de verdadeiras ausências em conjugação com as presenças, as possibilidades de avaliação são várias e regra geral robustas. Destas são exemplo o cálculo simples da percentagem de classificações correctas ou curvas Receiver Operating Characteristic (ROC). No entanto, a obtenção de um valor de desempenho utilizando apenas registos de presenças não é tão directa. Dando-se, por exemplo, o caso extremo de um modelo apresentar a totalidade dos seus valores como adequabilidade máxima, a percentagem de valores correctos, utilizando unicamente dados de presença, será sempre de $100 \%$, no entanto, aquele não terá qualquer tipo de utilidade pois a sua discriminação espacial é nula.

Aqui é proposta uma estratégia de avaliação com base na Área Mínima de Adequabilidade Máxima (AMAM). Este conceito, introduzido por Engler et al. (2004), diz respeito à superfície mínima obtida por um determinado limiar de probabilidade, acima do qual ainda se mantêm $90 \%$ de valores de validação correctos. Estes autores implementam-na como uma medida isolada, para ser 
utilizada em conjugação com outros factores, tendo em vista a comparação do desempenho de vários modelos. No entanto, como é referido por estes, a AMAM é, por si só, um aspecto de extrema importância, considerando que uma mesma percentagem de predições correctas terá tanto maior significado quanto menor for a área dada como de elevada adequabilidade. Derivando deste princípio foi elaborado um gráfico (fig. 4) que relaciona a área abrangida e o desempenho preditivo por limite de probabilidade para o presente modelo. É assim possível avaliar o comportamento preditivo do modelo com a variação da dimensão da área a que se destina o estudo.

O desempenho preditivo foi obtido através da validação cruzada do grupo de registos de presença deixados especificamente para este efeito $(n=30)$ e a carta contendo os valores de probabilidade de adequação do habitat.

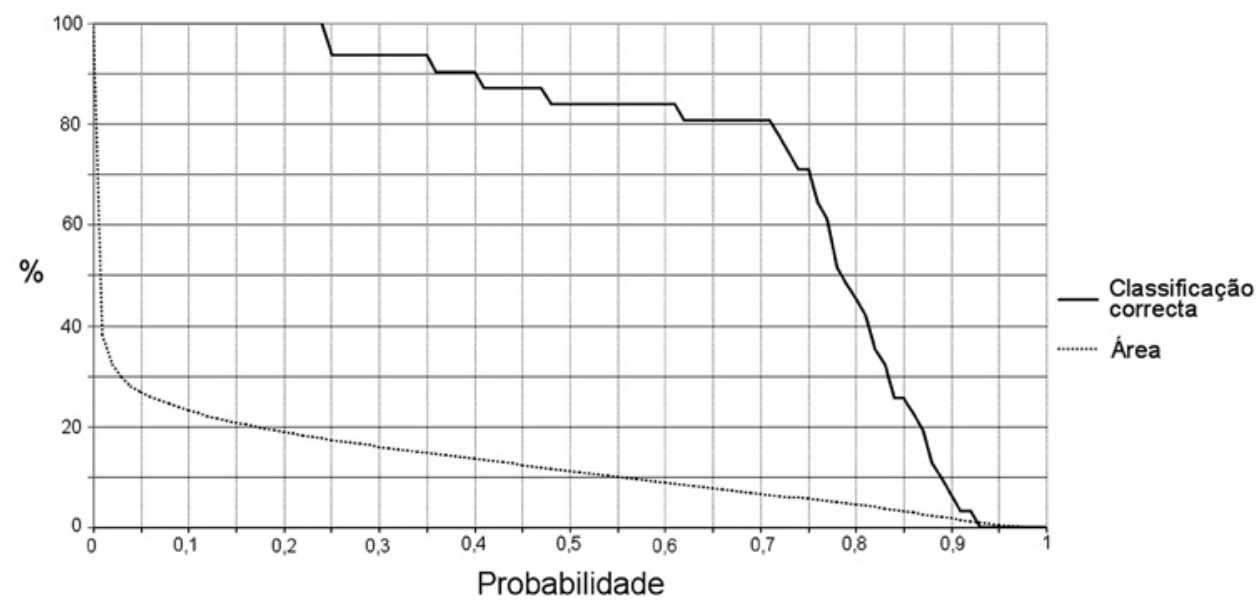

Fig. 4 - Percentagem de área e classificações correctas por limite de probabilidade de adequação Fig. 4 - Area percentage and correct classifications by limit of adequacy probability

Através da análise da relação é possível constatar que cerca de $75 \%$ da área de estudo apresenta probabilidade de adequação inferior a 0,1 , sendo a área situada acima de um limite de probabilidade de 0,5 correspondente a menos de $15 \%$ da área total. Os valores relativos à percentagem de classificações de presenças correctas apresentam, para este último limite (valor habitualmente utilizado para a separação entre o que se considera a ausência do fenómeno e a sua presença), um total de cerca de $85 \%$. Tendo em conta que este valor é obtido sobre uma área inferior a $15 \%$ da área total de estudo, pode-se considerar o modelo como tendo um desempenho preditivo elevado. Avançando até ao limite de 0,7 de probabilidade de adequação obtêm-se ainda cerca de $80 \%$ de classificações correctas para uma área correspondente a apenas $8 \%$ da área total. Este último 
limite foi considerado como o ideal para a separação entre áreas consideradas de adequabilidade baixa ou média e adequabilidade elevada.

\section{DISCUSSÃO DOS RESULTADOS}

A existência de elevados valores de classificação das presenças numa área de reduzida dimensão comparativamente à totalidade da área de estudo significa em grande parte que esta espécie foi encontrada em locais específicos onde existe uma maior adequabilidade de habitat. Esta situação de elevada especificidade de habitat parece indicar que a espécie se encontra num estado já próximo do equilíbrio com o ecossistema da área. Esta afirmação é ainda suportada pelo conhecimento da ocorrência da espécie na Europa Ocidental desde há largas décadas.

Através da análise da carta de adequabilidade de habitat (fig. 3) constata-se a existência de uma área alongada e paralela à costa, com probabilidade de adequação acima dos 70\%. Esta área compreende cerca de 276 hectares, o equivalente a cerca de $17 \%$ da área de estudo e distribui-se sobretudo ao longo do topo da Serra do Bouro, numa faixa compreendida entre os $150 \mathrm{~m}$ e os $1200 \mathrm{~m}$ de distância à linha de costa. A razão de probabilidade para esta variável ainda que inferior a $1(\exp \beta=0,996)$ não parece evidenciar uma maior adequabilidade com a proximidade do Oceano. No entanto, a reduzida extensão para o interior da área de estudo pode ter contribuído neste sentido. A própria disposição espacial das áreas de maior adequabilidade, paralelas à linha de costa e praticamente inexistentes em áreas de maior interioridade, parece suportar que este é, de facto, um factor que desempenha um papel importante na adequabilidade das áreas.

Pela indicação do modelo de que as áreas agrícolas são favoráveis à ocorrência da espécie $(\exp \beta=1,142)$ depreende-se sobretudo que o $C$. edulis tem vindo a beneficiar com o abandono agrícola. A diferença de cerca de três anos, entre a fotografia aérea de onde foi obtido o levantamento de usos e os levantamentos de presenças, acentuaram esta relação, uma vez que várias dessas áreas foram recentemente abandonadas, permitindo neste intervalo de tempo a sua ocupação pela espécie. A integração desta variável com distanciamento temporal e o resultado obtido relativamente a esta classe indiciam a pertinência da inclusão de factores que transmitam a dinâmica temporal, uma componente intrínseca das invasões biológicas. A permissividade relativa à cobertura do tipo herbáceo deriva também em parte desta situação, uma vez que muitos dos actuais locais com este tipo de cobertura correspondem a antigas áreas agrícolas. Os restantes locais que apresentam herbáceas como dominantes mas que não são áreas de elevada adequabilidade encontram-se sobretudo junto das arribas, em locais onde o Factor LS $(\exp \beta=0,599)$ se apresenta bastante elevado. A aparente contradição entre o tipo de contribuição do factor LS e do SPI $(\exp \beta=1,119)$, ambos indicadores da erosão, deve-se ao facto de este último apresentar uma maior inflação de valores para vários locais de declives suaves a médios. Estes 
locais correspondem sobretudo a encostas de vales pouco encaixados, em que o solo é maioritariamente arenoso devido ao transporte dos sedimentos mais finos, tendo-se aí registado várias ocorrências.

\section{CONCLUSÕES}

A modelação espacial de áreas de adequabilidade para um invasor é possível, recorrendo-se a técnicas de modelação de habitats potenciais para espécies nativas. Ao contrário do que acontece com registos de presença, os dados de pseudo-ausência permitem a aplicação de métodos de integração. A comparação de desempenhos é, no entanto, dificultada devido à reduzida generalização destes estudos, à dificuldade na obtenção de registos espaciais a escalas passíveis de representar com rigor a ocorrência destas espécies e, sobretudo, por as metodologias de avaliação não beneficiarem ainda de uma base comum. A inexistência de uma base cartográfica com estes registos a uma escala nacional, ou mesmo regional, contribui também para a própria negligenciação do problema. A dimensão temporal neste tipo de estudos deve ser tida em conta na interpretação dos resultados e, se possível, integrar a própria estratégia de modelação. Dentro das variáveis que usualmente integram este tipo de modelos o uso do solo pode ser um veículo privilegiado para a transmissão desta dinâmica.

Os resultados obtidos por estas metodologias permitem obter um traço geral dos potenciais impactes de espécies invasoras sobre o território e respectiva biodiversidade. É também possível expandir o conhecimento acerca dos factores ambientais que intervêm nos processos de invasão. Estas informações podem ser úteis para a definição de estratégias de monitorização, erradicação e mitigação de impactes das invasões biológicas.

\section{BIBLIOGRAFIA}

Alvarez-Arbesú R, Felicísimo A (2002) GIS and logistic regression as tools for environmental management: a coastal cliff vegetation model in Northern Spain. In Brebia C (ed.) Management Information Systems 2002: GIS and Remote Sensing. Wessex Institute of Technology: 215-224.

Araújo M, Guisan A (2006) Five (or so) challenges for species distribution modelling. Journal of Biogeography, 33(10): 1677-1688.

Arguimbau F, Sintes C, Martí C, Febrero O, Allés P, Gomila M, Barber M, Olives M (2004) Catàleg de la flora vascular de Menorca. Institut Menorquí d'Estudis, Maó.

Brotons L, Thuiller W, Araújo M, Hirzel A (2004) Presence-absence versus presence-only modelling methods for predicting bird habitat suitability. Ecography, 27(4): 437-448.

Cawley A (2003) Habitat modeling of invasive plants in Great Smoky Mountains National Park. Disertação de mestrado, University of North Carolina, Chapel Hill.

DiTomaso J, Healy E (2006) Weeds of California and other Western States University of California. Los Angeles. 
Elith J, Graham C, Anderson R, Dudik M, Ferrier S, Guisan A, Hijmans R, Huettmann F, Leathwick J, Lehmann A, Li J, Lohmann L, Loiselle B, Manion G, Moritz C, Nakamura M, Nakazawa Y, Overton J, Peterson T, Phillips S, Richardson K, Scachetti-Pereira R, Schapire R, Soberon J, Williams S, Wisz M, Zimmermann N (2006) Novel methods improve prediction of species distributions from occurrence data. Ecography, 29(2): 129-151.

Engler R, Guisan A, Rechsteiner L (2004) An improved approach for predicting the distribution of rare and endangered species from occurrence and pseudo-absence data. Journal of Applied Ecology, 41(22): 263-274.

Evans, A (2003) Economic dimensions of invasive species. Choices, Electronic Edition, 2. ${ }^{\circ}$ trimestre de 2003. [Acedido em 15 de Janeiro de 2008]. http://www.choicesmagazine.org/2003-2/20032-02.htm.

Ferrier S, Watson G, Pearce J, Drielsma M (2002) Extended statistical approaches to modelling spatial pattern in biodiversity in northeast New South Wales. I.Species-level modelling. Biodiversity and Conservation, 11(12): 2275-2307.

Ficetola F, Thuiller W, Miaud C (2007) Prediction and validation of the potential global distribution of a problematic alien invasive species - the American bullfrog. Diversity and Distributions, 13(4): 476-485.

Garson D (2007) Logistic regression. Statnotes: topics in multivariate analysis. North Carolina State University. [Acedido em 20 de Janeiro de 2008]. http://www2.chass.ncsu.edu/garson/pa765/ statnote.htm

Gilman E (1999) Fact Sheet FPS-109: Carpobrotus edulis. Environmental Horticulture Department of University of Florida. [Acedido em 20 de Janeiro de 2008]. http://hort.ifas.ufl.edu/shrubs/ caredua.pdf

Guisan A, Zimmermann E (2000) Predictive habitat distribution models in ecology. Ecological Modelling, 135(2): 147-186.

Haltuch A, Berkman A, Garton W (2000) Geographic information system (GIS) analysis of ecosystem invasion: exotic mussels in Lake Erie. Limnology and Oceanography, 45(8): 1778-1787.

Hastings A, Cuddington K, Davies K, Dugaw J, Elmendorf S, Freestone A, Harrison S, Holland M, Lambrinos J, Malvadkar U, Melbourne A, Moore K, Taylor C, Thomson D (2005) The spatial spread of invasions: new developments in theory and evidence. Ecology Letters, 8(1): 91-101.

Ilwis (2007) Ilwis 3.4 Open. $52^{\circ}$ North Initiative for Geospatial Open Source Software Gmb. Germany, Muenster. (software)

Juliano S, Lounibos L (2005) Ecology of invasive mosquitoes: effects on resident species and on human health. Ecology Letters, 8(5): 558-574.

Ministério do Ambiente (1999) Decreto-lei n. ${ }^{0}$ 565/99 de 21 de Dezembro. In Diário da República - I Série - A, 295: 9100-9114.

Pearce J, Boyce M (2006) Modelling distribution and abundance with presence-only data. Journal of Applied Ecology, 43(3): 405-412.

Perrings C, Dehnen-Schmutz K, Touza J, Williamson M (2005) How to manage biological invasions under globalization. Trends in Ecology \& Evolution, 20(5): 212-215.

Peterson A, Vieglais A (2001) Predicting species invasions using ecological niche modeling: new approaches from bioinformatics attack a pressing problem. BioScience, 51(5): 363-371.

Pimentel D, Lach L, Zuniga R, Morrison D (2000) Environmental and economic costs of nonindigenous species in the United States. BioScience, 50(1): 53-65.

Qiau-Ping W, Xiao-Guang C, Zhao-Rong L (2007) Invasive freshwater snail, China. Emerging Infectious Diseases, 13(7): 1119-1120. 
Sakai A, Allendorf F, Holt J, Lodge D, Molofsky J, With A, Baughman S, Cabin J, Cohen J, Ellstrand N, Mccauley D, O'Neil P, Parker I, Thompson J, Weller S (2001) The population biology of invasive species. Annual Review of Ecology and Systematics, 32: 305-332.

Schmalzer P, Hinkle C (1987) Species biology and potential for controlling four exotic plants (Ammophila arenaria, Carpobrotus edulis, Cortaderia jubata, and Gasoul crystallinum) on Vandenberg Air Force Base. Kennedy Space Center. NASA. [Acedido em 22 de Janeiro de 2008]. http://hdl. handle.net/2060/19880008764

Segurado P, Araújo M (2004) An evaluation of methods for modelling species distributions. Journal of Biogeography, 31(10): 1555-1568.

Tsoar A, Allouche O, Steinitz O, Rotem D, Kadmon R (2007) A comparative evaluation of presence only methods for modelling species distribution. Diversity and Distributions, 13(4): 397-405.

Underwood E, Klinger R, Moore P (2004) Predicting patterns of non-native plant invasions in Yosemite National Park, California, USA. Diversity and Distributions, 10(5): 447-459.

Williamson M (1996) Biological Invasions. Chapman \& Hall, London. 